\title{
Hsp90@chromatin.nucleus: an emerging hub of a networker
}

\author{
Ritwick Sawarkar ${ }^{1}$ and Renato Paro ${ }^{1,2}$ \\ ${ }^{1}$ Department of Biosystems Science and Engineering, ETH Zürich, Mattenstrasse 26, 4058 Basel, Switzerland \\ ${ }^{2}$ Faculty of Science, University of Basel, Klingelbergstrasse 50, 4056 Basel, Switzerland
}

The molecular chaperone heat-shock protein of $90 \mathrm{kDa}$ (Hsp90) stabilizes various proteins and occupies a central position in cellular networks. Hsp90 inhibitors are being tested in clinical trials as anticancer drugs. Recent studies have illuminated the unappreciated significance of this chaperone in chromatin transactions and this review focuses on its role in gene expression. By comparing the different mechanisms by which Hsp90 orchestrates transcriptional regulation, the review outlines the contributions of this function to the cellular and organismal phenotype. We chart exciting avenues for research to gain comprehensive insights in the chaperone's importance in cellular physiology, thereby presenting novel opportunities for therapeutic intervention.

The cellular and nuclear context of the Hsp90 chaperone Molecular chaperones help nascent polypeptides acquire their correct conformations, thereby minimizing their aggregation in the protein-rich conditions inside the cell. Hsp90 is a specialized chaperone that stabilizes numerous important proteins and helps in the assembly of multimeric complexes [1-3]. With the aid of several co-chaperones and driven by its own ATPase activity, Hsp90 dynamically associates with target proteins (termed clients), keeping them in a stable or a stimulus-responsive conformation (Box 1). The clientele of Hsp90 comprises signaling proteins as well as transcription factors among many others, placing the chaperone as one of the few wellconnected hubs in molecular networks. Hsp90 is thought to play a crucial role in areas as diverse as development and homeostasis, cancer pathogenesis, and organismal evolution $[4,5]$.

The nuclear form constitutes about 2-3\% of total cellular Hsp90 and comparatively little attention has been paid to the specific tasks Hsp90 performs at chromatin. Several recent studies, however, have revealed a profound regulatory involvement of Hsp90 and its co-chaperones in basic nuclear processes. These range from telomere biology and DNA replication to transcription [6-9]. In this review, we explore the roles this versatile chaperone plays in the process of gene expression. We begin by collating data from various studies over the past few years to synthesize a picture of the life of Hsp90 in the nucleus. We then discuss how this chaperone integrates within the emerging framework of transcriptional regulation and epigenetic

Corresponding author: Paro, R. (renato.paro@bsse.ethz.ch)

Keywords: transcriptional regulation; chromatin; cancer; epigenetics; evolution. gene control. Finally, we evaluate the roles of this chaperone in medicine and evolution with the new insights from chromatin-associated Hsp90, charting future avenues for research.

\section{Hsp90 in transcriptional regulation}

Hsp90 has long been known to interact with chromatin, partly by binding to histones $[10,11]$. That the chaperone is found on heat-shock puffs on polytene chromosomes (see Glossary) of Drosophila underscored the functional relevance of Hsp90 in transcription [12]. Studies over the past decade, employing three independent approaches, have illuminated several fascinating aspects of gene expression controlled by Hsp90.

\section{Lessons from model genes and transcription factors}

In the first approach, studying model genes, a large repertoire of molecular mechanisms has been deciphered relating Hsp90's chaperone activity to some of the well-known transcriptional regulators (Figure 1). Chromatin-associated

\footnotetext{
Glossary

Argonaute 2 (Ago2) and RNAi: Argonautes form catalytic components of the RNA-induced silencing complex (RISC), which mediates the effect of small RNA, in a process called RNA interference (RNAi) [71]. The small RNAs along with the RISC complex exert their influence on the stability, translation, or expression of transcripts.

Chaperone-mediated autophagy: a process by which individual soluble proteins are recognized and targeted by the chaperones Hsp70/Hsp90 to lysosomes for degradation [48]. The target proteins are unfolded as they enter the lumen of lysosomes. Hsp70 plays a critical role in identifying substrates with a typical pentapeptide motif.

Chromosome puffs: in the nuclei of Drosophila salivary gland cells, chromosomes endoreplicate several times and align with each other lengthwise. This results in large chromosomes, termed polytene chromosomes, that can be visualized by light microscopy. An actively transcribing gene on a polytene chromosome appears as a decondensed bulge at the chromosomal locus. This is termed a puff and a protein associated with transcriptional elongation typically appears to stain uniformly across the puff.

Polycomb group (PcG) and Trithorax group (TrxG) proteins: these are chromatin proteins responsible for transcriptional memory through cell divisions. PcG protein form well-defined complexes and bind promoters of developmentally important genes [72]. By specifically modifying chromatin, the PcG heritably represses target genes, like the Hox genes, such that genes that are inactive during early embryonic tissues remain repressed throughout development [73]. Trimethylation of lysine 27 of histone $\mathrm{H} 3(\mathrm{H} 3 \mathrm{~K} 27 \mathrm{me} 3)$ and ubiquitination of lysine 119 of histone $\mathrm{H} 2 \mathrm{~A}$ (H2AK119ub) are two of the epigenetic marks set by PcG and are typically associated with gene inactivity. TrxG proteins antagonize this repression and keep lineage-specific genes active through cell division by trimethylating lysine 4 of histone $\mathrm{H} 3$ (H3K4me3) [74]. The mechanism by which PcG and TrxG proteins act at chromatin is not completely understood, but it involves chromatin compaction and retention of pol II from entering the elongation mode [42]. Many PcG/TrxG proteins are implicated in the pathogenesis of cancer.
} 


\section{Box 1. The molecular mechanism of Hsp90-mediated} chaperoning

Hsp90 protein can be divided into an $\mathrm{N}$-terminal ATP-binding domain, a $\mathrm{C}$ terminus harboring a dimerization motif, and middle client/co-chaperone-binding domain [66]. Hsp90 exists as a dimer and cycles between two states - a 'closed' state, wherein ATP binding leads to close association of the N-terminal domains, and an 'open' state when the N-terminal domains of the two monomers are farther away from each other [67]. This chaperone cycle accommodates client loading and, through repeated binding of labile pockets of the client protein, Hsp90 stabilizes and keeps it in a responsive state. The molecular details of this process are being gathered through meticulous structural and biophysical experiments and a full picture may soon emerge. Several co-chaperones help Hsp90 achieve its full potential [68]; Aha1, for example, stimulates the ATPase activity of Hsp90. Cdc37 potentiates the interaction of the chaperone with specific kinase substrates [69]. In addition to helping specific client proteins fold, Hsp90 plays a vital role in the building of many multiprotein complexes, such as $26 \mathrm{~S}$ proteasome and telomere complex [70]. The chaperone, aided by co-chaperones such as Pih1 and Tah1, takes part in the stepwise assembly of these complexes, including RNA polymerase II.

Hsp90 affects the outcome of gene expression control by different means depending on the context. These experiments have suggested that Hsp90 does not act as a general repressor or an activator of transcription, but rather chaperones different proteins in a gene-specific way. It is required for release of glucocorticoid receptors from chromatin after the hormonal ligands are taken away [13]. p23 may aid Hsp90 in the process of disassembly of steroid receptormediated active transcriptional complex at chromatin [14]. By stabilizing a repressor called BCL-6 at promoters, Hsp90 acts to keep target genes silent in a type of B-cell lymphoma [15]. Hsp90 inhibition in these cells results in derepression of many of these targets, including the tumor suppressor p53. Hsp90 can also activate gene expression by stabilizing and activating either transcription factors such as Sp1 [16], signal transducer and activator of transcription 5 (STAT5) [17] and MyoD1 [18] or epigenetic regulators like Trithorax [19]. Curiously, Hsp90 along with STAT5 binds the promoter of the MYC gene in a human leukemia cell line. Hsp90 inhibition causes downregulation of MYC in these cells, suggesting a STAT5-dependent activator function of Hsp90 in this context [17]. Similarly, Hsp90 inhibition in Drosophila cells results in downregulation of Hox target genes concomitant with degradation of Trithorax, an activator of gene expression [19]. In other cases, this activating role is mediated by the Hsp90-dependent function of the histone-modifying enzyme SET and MYND domain containing 3 (SMYD3) [20]. Additionally, Hsp90 and Hsp70, another major chaperone, may also contribute to gene induction by removing nucleosomes, as shown for galactose genes in budding yeast [21]. Overall, it appears that Hsp90 wears different hats at different promoters - it may sometimes be required for gene activation and at other times for gene repression. It must be noted, however, that most studies analyze the transcriptional output of target genes after several hours or days of Hsp90 inhibition. Given the versatility of this chaperone in signaling networks in the cytosol, such long durations may confound interpretations attributing specific transcriptional functions to Hsp90.

\section{Hsp90 interactome studies}

As another approach to identify the transcriptional roles of Hsp90, interactome analyses or genetic screens have coincidentally pointed to the possible roles of the chaperone in gene expression. A regularly updated list of all Hsp90 interactors is maintained online (http://www. picard.ch/Hsp90Int/index.php) and a comprehensive understanding from such global analyses has been recently reviewed [22]. Many of these interactions are independently identified in different organisms and strongly suggest collaboration between Hsp90 and the transcriptional apparatus (a subset is listed in Table 1). Curiously, Hsp90 inhibition leads to several changes of chromatin; heterochromatin protein 1 (HP1) levels increase, whereas histone acetyltransferase 1 and the histone arginine methyltransferase PRMT5 decrease [23]. How this alters the transcriptional output of inhibitor-treated cells remains an open question. Besides the proteins directly involved in chromatin operations, two categories of proteins stand out: RNA processing/splicing proteins and DNA replication/damage-response proteins [24-27]. The diversity of Hsp90's clientele may allow this chaperone functionally to couple distinct processes such as replication, DNA damage response, transcription, nuclear architecture, and splicing [9,28]. Moreover, the importance of the chaperone in the interaction between steroid receptors and nuclear pores [29] may suggest mechanistic links between nuclear Hsp90, transcription, and nuclear pores [30].

\section{Global chromatin studies}

The third approach to uncover the transcriptional function of Hsp90 employed a systems biology-driven hypothesis coupled to experimental validation. A global and high-resolution chromatin-binding profile of Hsp90 using chromatin immunoprecipitation sequencing (ChIP-seq) of cultured fly cells allowed direct comparison of chaperone binding with other transcriptional features [31]. Three general principles of transcriptional regulation by Hsp90 became clear (Figure 2). First, the chaperone accumulates close to the transcription start sites (TSSs) of onethird of all protein-coding genes and several miRNAcoding genes. Second, the Hsp90-target genes have one regulatory feature in common - they all exhibit the paused state of RNA polymerase II (pol II) (Box 2 and Figure 2). Third, Hsp90 inhibition releases the pause within minutes, causing robust upregulation of many of the target genes. Evidently, one of the factors required for pol II pausing, namely the Negative Elongation Factor (NELF) complex, is bound and stabilized by Hsp90 at promoters. Thus, the genome-wide approach combined with quantitative analysis of immediate transcriptional effects paved the way for detailed molecular understanding of the distinctive functions of Hsp90 at chromatin. Interestingly, this study based primarily in Drosophila brought together hitherto unlinked observations regarding HIV. Independent studies have demonstrated that the promoter of HIV provirus shows pol II pausing [32] and Hsp90 recruitment [33]. It can thus be speculated that the chaperone controls replication of this retrovirus by influencing pol II pausing. 


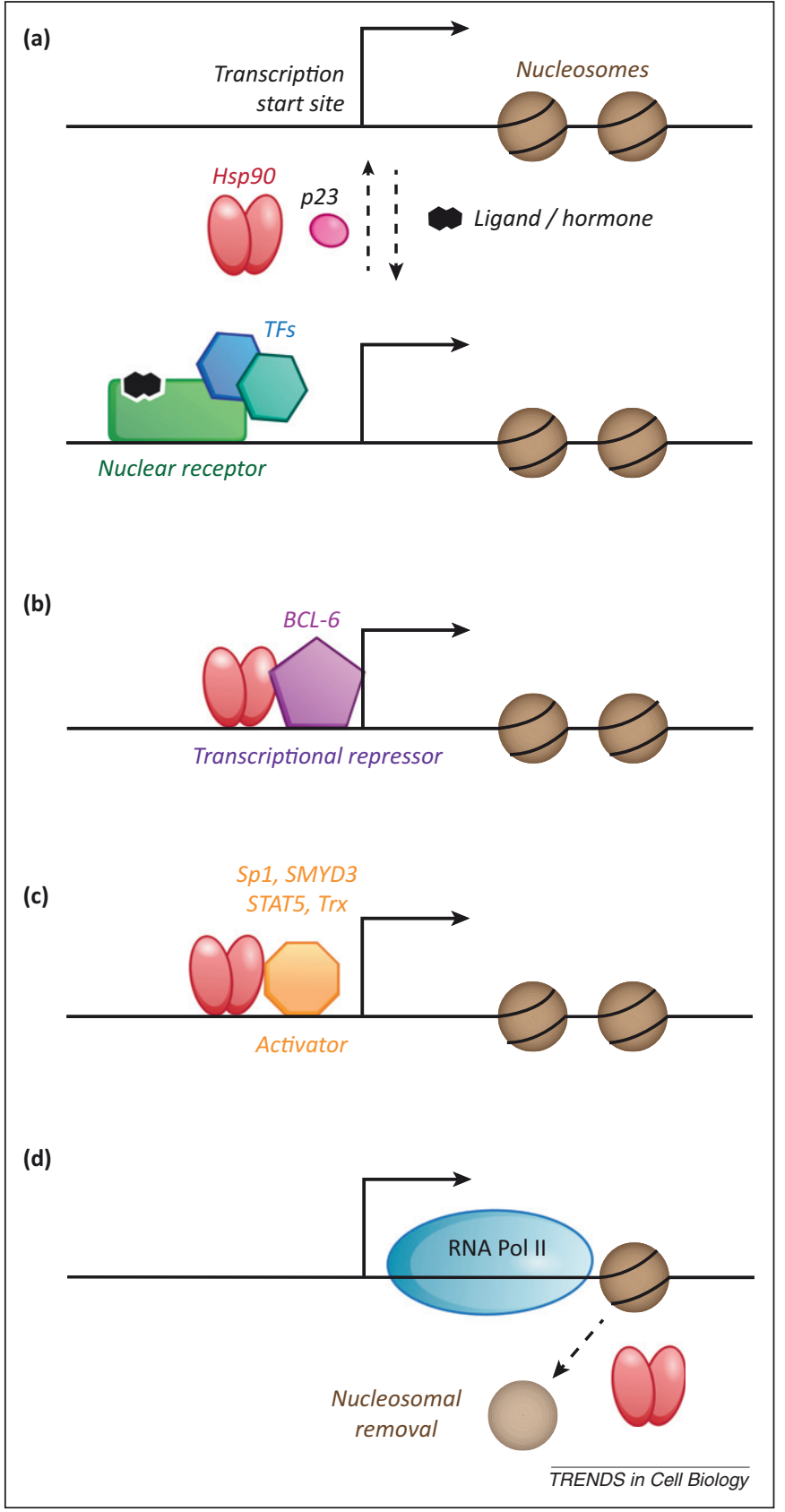

Figure 1. The diverse roles of heat-shock protein of $90 \mathrm{kDa}(\mathrm{Hsp} 90)$ in gene regulation. The gene regulatory region, transcription start site (TSS), and downstream nucleosomes are depicted schematically. Hsp90 along with p23 is involved in disassembling the nuclear receptor complexes formed at target promoters on stimulation with ligand/hormones (a). Hsp90 chaperones various proteins that act as either transcriptional repressors (b) or activators (c), depending on the cell type and target genes. Hsp90 also aids in removing nucleosomes from induced genes in yeast, facilitating transcription by RNA polymerase II (pol II) (d). The specific proteins/complexes that are influenced by $\mathrm{Hsp} 90$ are depicted. It should be noted that arrows indicate the position of the TSS and do not convey any information about the gene expression status. Abbreviations: TF, transcription factor; STAT5, signal transducer and activator of transcription 5; SMYD3, SET and MYND domain containing 3; Trx, Trithorax (the fly ortholog of mammalian mixed lineage leukemia [MLL]).

\section{Functional interactions between Hsp90 and transcription machinery at chromatin}

The role of Hsp90 in the pausing process is conceptually similar to the way by which the chaperone maintains steroid receptors in a hormone-responsive state [34]. Hsp90's ability to bind transcriptionally active puffs on polytene chromosomes $[12,19]$ may suggest that the chaperone accompanies the elongating form of pol II over the gene body. Additionally, Hsp90 complexes on inhibition become enriched with the core transcription machinery [35], emphasizing the importance of Hsp90 in transcription beyond pausing. Given that Hsp90 plays a critical role in building the RNA pol II complex in cytosol [36], the chaperone may structurally assist paused or elongating pol II complex. Additionally, Hsp90 may actively couple pol II with the splicing machinery. An exciting avenue for research is the regulation of Hsp90 itself during the transcriptional cycle, at paused promoters and over active gene bodies. It is likely that the chaperone is subject to the same post-translational modifications that influence pol II and chromatin factors. For example, positive transcription Elongation Factor b (pTEFb) with its Cdk9 kinase may phosphorylate Hsp90 along with other pausing factors (Figure 2) and this step may be necessary for efficient gene activation. Additionally, Hsp90 may be acetylated or methylated by a myriad of chromatin-modifying enzymes located at promoters. Cytosolic isoforms of Hsp90 are known to be regulated by post-translational modifications [37] and these may also influence the chromatin-associated form. In this regard, it is noteworthy that phosphorylation of the chaperone correlates with its nuclear localization [38] and stability [39]. How these modifications affect the residence time of the chaperone at chromatin is an important question to gain deeper insights in the chaperone cycle at this subcellular location. Whereas the Drosophila genome encodes only one isoform of Hsp90, mammalian cells express two related proteins $-\mathrm{Hsp} 90 \alpha$ and $\beta$. The chromatin distribution and specific nuclear complexes of the two isoforms are unknown, but will be significant in providing a comprehensive understanding. This is especially relevant in the light of the finding that some nuclear events are exclusive to one isoform [38].

\section{Chromatin association of Hsp90 and heat shock}

One question that needs immediate attention is what recruits Hsp90 to chromatin at specific promoters, as indicated by genome-wide studies. Given that Hsp90 does not show sequence-specific DNA-binding ability, it may require other factors to localize. Earlier studies analyzing a small set of loci have argued for specific transcription factors as clients recruiting the chaperone to distinct loci (see above). In addition, a more generic factor such as RNA pol II or the pausing factor NELF complex are likely to be involved in this process and further experiments are required to address this issue.

Inhibition of Hsp90 is typically thought to elicit a transcriptional response similar to that of elevated temperature. Indeed, both activate heat-shock genes, albeit to different extents. However, at chromatin these two processes may operate with opposite mechanisms. Heat-shock conditions cause relocalization of pol II from almost all genes to heat-shock loci, such that the amount of elongating pol II decreases in the gene bodies of most genes [40]. This results in a rapid decrease in the fresh transcription of all genes except heat-shock genes. However, Hsp90 inhibition causes an increase in elongating pol II in many of its target genes that are upregulated within minutes. The 
Table 1. A subset of transcription-related proteins interacting with Hsp90

\begin{tabular}{|c|c|c|}
\hline Protein name & Remarks; function & Refs \\
\hline RNA polymerase II associated protein 3 (RPAP3) & Interacts with Hsp90; involved in RNA pol II assembly & {$[35,75,76$} \\
\hline RNA pol II polypeptide E & Interacts with Hsp90 & {$[25,35,75$} \\
\hline CCR4-NOT transcription complex, subunit 6 & Interacts with Hsp90; core transcriptional regulator & [35] \\
\hline MED7 (Mediator complex subunit 7) & $\begin{array}{l}\text { Interacts with Hsp90; cofactor required for transcription and SP1 } \\
\text { activation }\end{array}$ & [24] \\
\hline $48 \mathrm{kDa}$ TATA box-binding protein-interacting protein (RUVBL2) & Interacts with Hsp90; involved in chromatin remodeling & [76] \\
\hline TATA box binding protein (TBP) & Interacts with Hsp90 & [24] \\
\hline HP1gamma/chromobox protein homolog 3 (Cbx3) & Forms aggresome in response to radicicol & {$[23,27]$} \\
\hline Nuclear pore complex protein (NUP155) & Genetic interaction with Hsp90 & [78] \\
\hline Nucleoporin p54 (NUP54) & Forms aggresome in response to radicicol & [27] \\
\hline Suppressor of Ty's 2 (SPT2)/EXA1 & Interacts with Cdc37; involved in RNA polyadenylation & [80] \\
\hline VPS71/SWR1 complex component 6 (SWC6) & Interacts with Cdc37; involved in chromatin remodeling & [80] \\
\hline
\end{tabular}

mechanism by which heat shock causes depletion of pol II at gene bodies is unclear, but may involve nucleosomal turnover [41]. Whether Hsp90 contributes to this process is an important mechanistic question. In this respect, it is interesting to note that Hsp90 binds to many heat-shock genes before the heat shock occurs [31], as most exhibit paused pol II. Heat-shock factor (HSF), which activates these genes on stress, is a known client of Hsp90 and the two may collaborate at sites of gene expression during heat shock - a possibility that is yet to be tested.

\section{Hsp90 enters the world of epigenetics}

Several well-characterized target genes that are upregulated on Hsp90 inhibition, such as MYC and TP53, are subject to exquisite epigenetic control. Chromatin repressors involved in transcriptional memory called Polycomb group (PcG) proteins co-occupy numerous Hsp90 target promoters. At several loci, Hsp90 functions in the context of the repression by PcG proteins. How the environmentsensitive chaperone network interacts with the epigenetic machinery in influencing the status of RNA pol II is only beginning to be understood. Considering that the target genes are activated within minutes after Hsp90 inhibition, it will be interesting to study the fate of these repressors and the associated chromatin marks at these loci; for example, whether H3K27 demethylation and H2AK119 deubiquitination resulting in the loss of these repressive marks parallel Hsp90 inhibition kinetics. Also, it is unclear whether PcG proteins are delocalized from these promoters and, if so, what mechanisms operate in that process. Could client proteins such as BCL-6 repressor act to target PcG at Hsp90-target promoters? Because PcG and Trithorax group (TrxG) proteins are involved in retaining the state of gene expression [42], will the target genes continue to be activated after transient inhibition of Hsp90? Are PcG proteins reloaded at the appropriate loci after reinstating Hsp90 activity? As mentioned above, longer inhibition of Hsp90 results in downregulation of a few Hsp90 target genes, presumably owing to degradation of Trithorax (TRX), an activator of gene expression. How does the same chaperone control these opposing activities at gene promoters? Many of these questions can be answered by careful analysis of epigenetic events before, during, and after Hsp90 inhibition. However, one likely difficulty in obtaining accurate answers is the inability to assess these events at the single-cell level, because the population average of several epigenetic events may not be a true representation of the temporal changes occurring at chromatin within a single cell.

Many proteins besides PcG/TrxG influence pol II pausing/elongation control. Recently, Argonaute 2 (Ago2) was shown to localize to promoters with paused pol II in Drosophila cells and to modulate the activity of pol II [43]. It is known that Hsp90 is involved in the assembly of the Argonaute-small RNA effector complex in cytosol $[44,45]$. Thus, it is highly likely that a promoter-associated chaperone is required for the proper functioning of Ago2 and the consequent regulation of pol II function as well. It will be interesting to analyze the functional interaction between Hsp90 and Ago2 at promoters, to determine how they collaborate in deciding the fate of pol II.

Hsp90, in conjunction with Hsp70, has been recently shown to play a role in histone turnover in cytosol via chaperone-mediated autophagy (CMA) [46]. Regulated turnover of nucleosomal histones is a hallmark of promoters and regulatory regions of chromatin [47] and chaperones like Hsp90 and Hsp70 are important in this process [21]. Hsp90 bound to promoter regions may thus be instrumental in coupling nucleosome removal and their degradation by CMA. Although autophagy is unlikely to occur inside the nucleus, the chaperone could ferry the histones to the cytosol for further processing, thus keeping them distinct from fresh histone pools. Indeed, several transcription factors are substrates for CMA [48], underlining the 


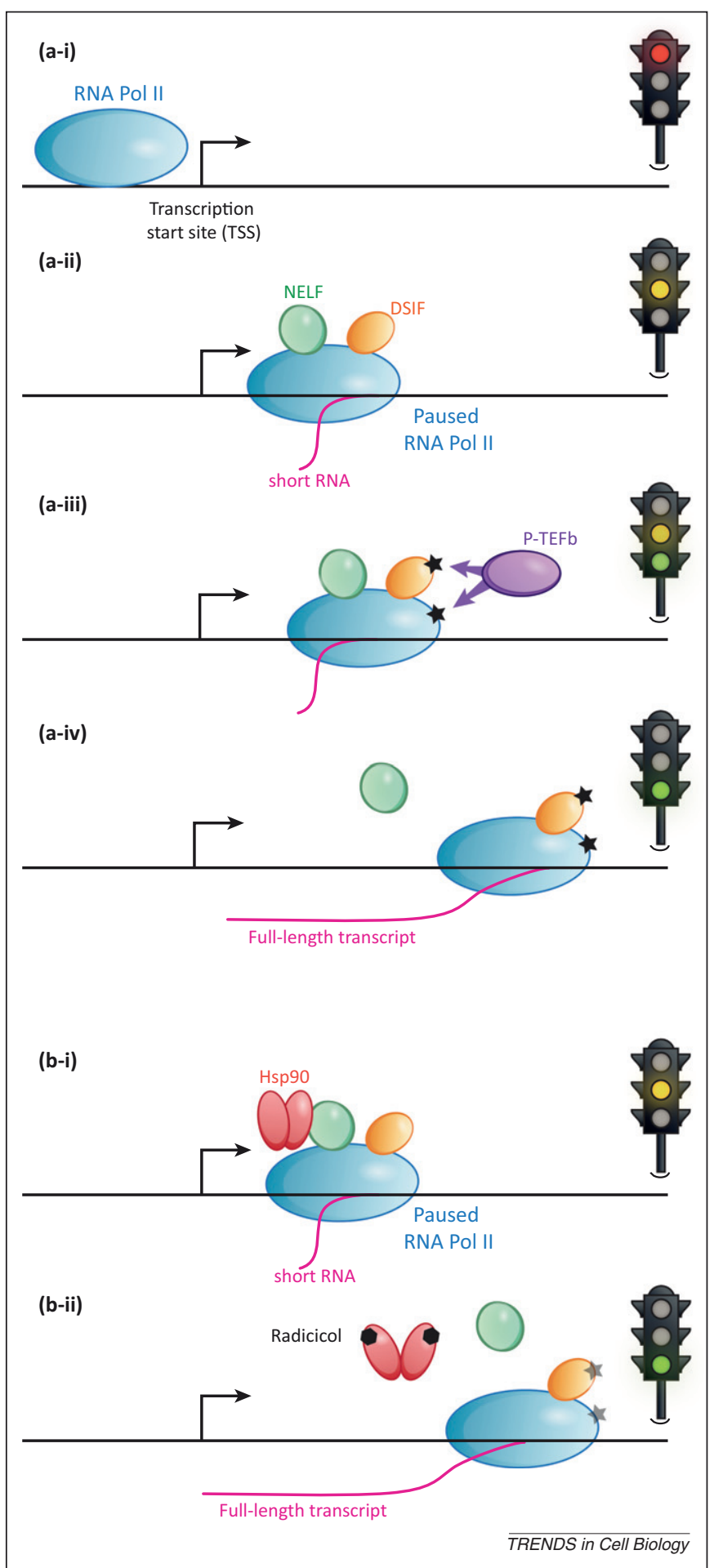

Figure 2. The process of pausing of RNA polymerase II (pol II) and its regulation by heat-shock protein of $90 \mathrm{kDa}$ (Hsp90). The RNA pol II complex is assembled upstream of the transcription start site (TSS) (a-i) and then in many cases transcribes a short piece of RNA until it halts or pauses under the influence of the Negative Elongation Factor (NELF) and DRB sensitivity-inducing factor (DSIF) complexes (a-ii). Positive transcription Elongation Factor b (pTEFb) recruited by transcription factors (TFs) phosphorylates DSIF and pol II (depicted by stars) (a-iii), allowing the latter to synthesize a full-length transcript (a-iv). The traffic lights on the right depict the state of RNA pol II and transcription: red, inactive pol II; yellow, paused pol II; green, actively transcribing pol II. Hsp90 preferentially localizes to promoters that exhibit pol II pausing (b-i). Inhibition of the chaperone leads to a decrease of chromatin-associated NELF, release of pausing, and production of a full-length transcript (b-ii). It is unclear whether pol II and DSIF are phosphorylated by $\mathrm{pTEFb}$ in this case (depicted as gray stars).

\section{Box 2. RNA polymerase II pausing and elongation control}

Recent studies indicate that about one-third of all genes in fly and mammalian cells exhibit RNA pol II molecules that are recruited to promoters but do not enter elongation mode immediately [81]. After initiation at transcription start sites, Pol II transcribes for about 40 100 bases and then pauses, making only a short RNA (Figure 2). Two complexes play an instructive role in this process: the Negative Elongation Factor (NELF) complex and the DRB sensitivity-inducing factor (DSIF) complex [82]. At a molecular level, how pausing is precisely orchestrated is not yet understood. To activate a paused gene, pTEFb (comprising Cyclin T1 and CDK9) is recruited to promoters, where it phosphorylates pol II, NELF, and DSIF [83]. This releases NELF from chromatin and allows pol II to extend in the gene body, making a full-length transcript. This was first reported for the Hsp70 gene in Drosophila and for the MYC gene in mammalian cells. The pausing process is thought to keep a gene in a ready-to-induce form such that the rate-limiting steps of pol II assembly at promoters are avoided. Alternative possibilities have also been speculated on recently [84].

importance of this mode of turnover that is common to chromatin proteins and possibly nucleosomes.

\section{The role of chromatin-associated Hsp90 in biomedicine and evolution}

Hsp90, genetic variation, and evolution

Hsp90 is proposed to act as a buffer and hence was termed a capacitor of genetic variation, aiding rapid evolution (Box 3 ). It is thought that the buffering capacity of $\mathrm{Hsp} 90$ is directed toward variation occurring in client protein sequences [4]. This hypothesis stems from decades of work on Hsp90 client interactions in cytosol. However, many studies have argued that morphological evolution also occurs by changes in the space and time of expression of proteins and not only by changing protein sequences per se [49,50]. In other words, cis-regulatory variation that affects spatial and temporal patterns of developmental gene expression is speculated to be an important substrate for natural selection to act on. The finding that Hsp90 is localized to chromatin at the promoters of several developmentally important genes thus suggests another intriguing possibility. The chaperone may buffer genetic variation at target promoters; that is, at sequences that determine the time and place of expression of developmentally relevant genes (Figure 3). Mechanistically, this can be achieved if Hsp90 controls the pausing of pol II despite underlying variation at promoter sequences. It is likely that Hsp90 dynamically stabilizes the NELF complex to a similar extent irrespective of minor alterations in the DNA sequence affecting sequence-specific regulatory proteins. Although this has not been substantiated, such predictions can be tested with reconstituted systems of pol pausing and regulated release thereof. It should be noted that causative polymorphisms of Hsp90-contingent traits have been mapped to both coding and regulatory genomic regions in natural yeast strains [51]. Whether this holds true for metazoan populations that have distinct developmental programs encoded in the genome remains an exciting area of future research.

In addition to genetic variation, Hsp90 also buffers epigenetic variation in conjunction with Trithorax [52]. Although the true nature of this variation remains elusive, it may stem from the promoters themselves, since both Trithorax and Hsp90 co-occupy several promoters [19]. 


\section{Box 3. The proposed role of Hsp90 in evolution}

Inhibition of Hsp90 in developing organisms such as Drosophila and Arabidopsis results in phenotypic differences in adult structures $[85,86]$. This emergent phenotypic variation was thought to have resulted from pre-existing genetic variation. This led to the hypothesis that, under normal conditions, Hsp90 minimizes the effects of genetic variation such that different variants produce one/ few canalized phenotypes. On stress, when the limited pool of Hsp90 is drawn out of this buffering function, genetic variation manifests in phenotypic variation (Figure 3 ). It is this variation on which natural selection can act, resulting in the emergence of novel phenotypes suited to the stressful conditions. A corollary to this logic is that Hsp90 allows accumulation of genetic variation by reducing its consequences on fitness, thus acting as a 'capacitor' [87]. In other words, Hsp90 uncouples genotype from phenotype and in doing so perpetuates genetic variants that may otherwise have been selected out. It has been difficult to prove all of the tenets of this hypothesis in a metazoan population, but recent work on naturally occurring yeasts has garnered some support for the hypothesis [51]. However, alternative possibilities for the source of phenotypic variation have also been suggested. In flies, the phenotypic variation need not stem from pre-existing genetic variation, but epigenetic variation and transposon activation on Hsp90 depletion may also contribute $[52,88,89]$.

Given that PcG/TrxG systems can epigenetically inherit the state of gene expression [53,54], Hsp90-buffered epigenetic variation may be inherited through the germline favoring rapid propagation of selectable phenotypic traits. Several recent reports attest to the possibility of such epigenetic inheritance, even in mammals [55], but a clever experimental design is required to settle this issue.

\section{Hsp90 and cancer treatment}

The singularly important contribution of Hsp90 inhibitors in the biomedical field is in the treatment of various solid tumors and leukemia $[5,56,57]$. More than a dozen Hsp90 inhibitors are in different phases of clinical trials [58]. Although the precise mechanism by which the inhibitors selectively affect cancer cells is unclear, it is thought that most oncoproteins depend on Hsp90 for their deadly performance. However, the finding of chromatin-associated Hsp90 requires careful consideration in this medically relevant context. First, critical drivers of several cancers are Hsp90 client nuclear proteins that cause aberrant gene expression. Hsp90 binds Trithorax/Mixed Lineage Leukemia (MLL) at chromatin [19] and fusion of MLL with members of the transcriptional elongation complex leads to several types of leukemia [59]. The BCL-6 transcriptional repressor that causes diffuse large B-cell lymphoma is a known client of Hsp90 at promoters of genes such as TP53 [15]. Second, HSF was recently shown to have a malignant cancer-specific chromatin profile affecting a distinct set of genes depending on the stage of cancer [60]. This factor is a well-known client of Hsp90 and the two proteins share a large set of chromatin targets in fly cells [31]. Third, Hsp90 itself targets and regulates promoters of MYC, TP53, and many miRNAs involved in apoptosis and cellular proliferation in human as well as fly cells [15,17,31]. Fourth, histone deacetylase (HDAC) inhibitors have a synergistic effect on reducing cancer when combined with classical Hsp90 inhibitors [61]. Although not directly demonstrated,

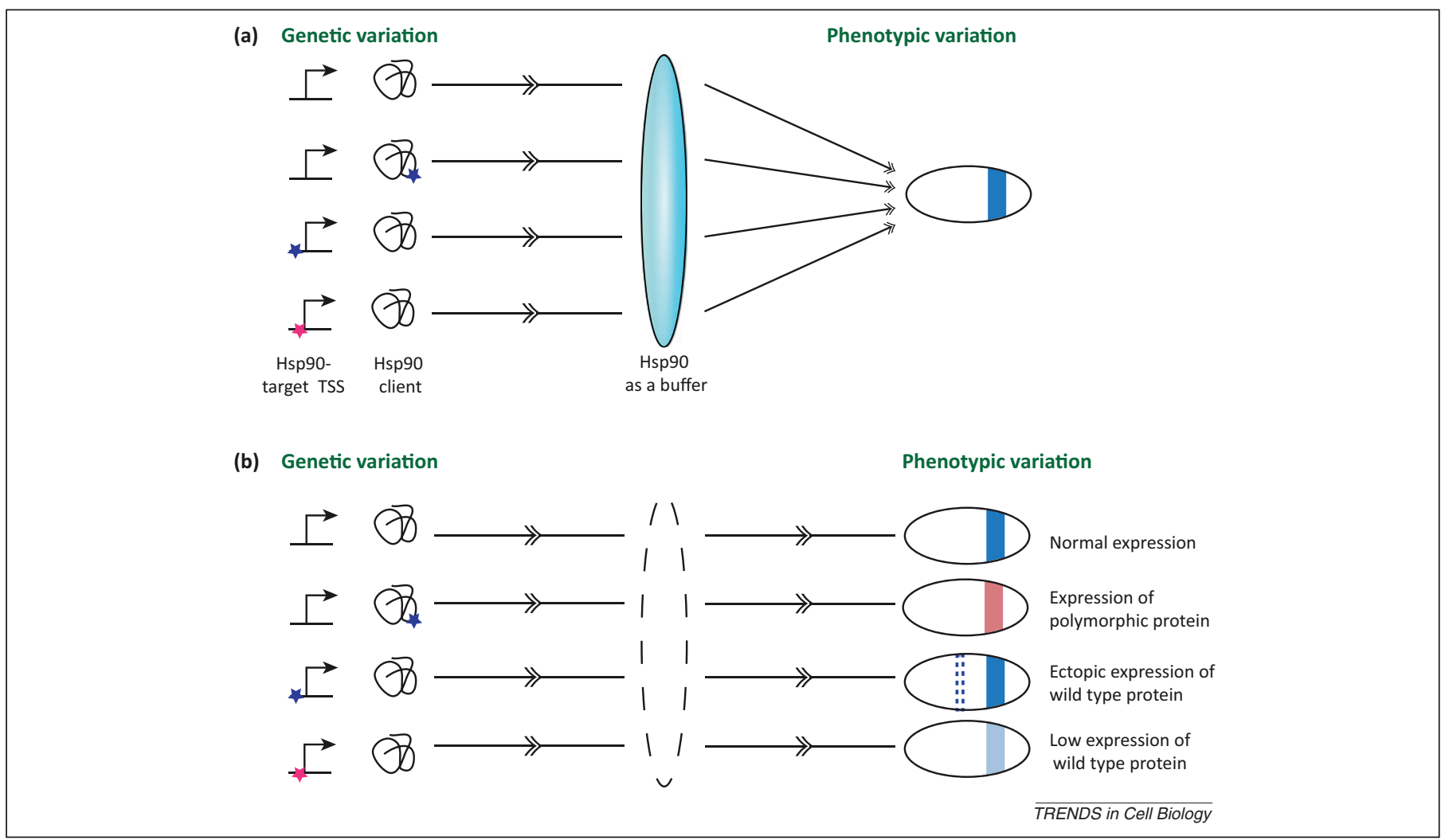

Figure 3. The proposed role of heat-shock protein of $90 \mathrm{kDa}(\mathrm{Hsp} 90)$ in genotype-phenotype relation. Hsp90 acts similarly to a convex lens in canalizing different genetic variants to produce one/few phenotypic variants (a). The genetic variants are depicted as colored stars at transcription start sites (TSSs) (cis-regulatory variants) or in Hsp90 client proteins (structural variants). A fly embryo with expression of a developmental gene (blue stripe on an embryo) is shown here as an example of a phenotype. When Hsp90 function is compromised (b), the genetic variants - TSS-associated or in protein sequences - may manifest as phenotypic variants. These are depicted as ectopic or low expression of wild type proteins or expression of variant polymorphic proteins. Natural selection may favor one over the others, selecting for that phenotypic trait and facilitating rapid evolution. 
it is possible that both inhibitors collaborate at the level of gene expression in reducing the aberrant expression of genes responsible for carcinogenesis. Finally, Hsp90 inhibitor-treated cancer cells develop resistance to drugs primarily by mounting a heat-shock response [62]. Given that Hsp90 associates with the promoters of most heat-shock genes, it is possible that the chromatin-associated chaperone is crucial for development of this resistance. All these issues beg for reevaluation of strategies to combat cancer by inhibiting the molecular chaperone. Separate consideration of nuclear and cytosolic pools of Hsp90 may be a more rational and objective way to approach the issue. Whereas some cancers may rely on nuclear Hsp90 (such as those driven by MLL fusion proteins), others may depend only on cytosolic pools. Inhibition of nuclear Hsp90 in the latter case may thus bring about toxic side effects that could be avoided by a more strategic treatment regimen. Development of drugs that specifically target the chaperone in distinct subcellular compartments will be the first step toward more efficient treatment. Recent research on targeting the mitochondrial pool of Hsp90 attests to the plausibility of such an approach [63]. How can the cytosolic and nuclear pools of the chaperone be independently inhibited? Making use of activating enzymes such as esterases that are specific to a compartment may be one approach. Tapping the potential of the selective permeability of the nuclear membrane might be another way. A more biologically inspired method would be to target the nucleusspecific co-chaperones, if any, that Hsp90 employs. Hsp90 is known to have an altered interactome in cancer cells, because they contain a higher-ordered multichaperone complex [17]. A chromatin-based Hsp90 interactome in cancer and normal cells may identify convenient drug targets. Additionally, promoter-specific post-translational modifications of Hsp90 may render the nuclear chaperone more susceptible to pre-existing drugs compared with its cytosolic counterpart. Moreover, nuclear entry of the chaperone might itself be a possible target step, because chaperones like Hsp70 require distinct protein networks for nuclear entry [64]. Overall, a thorough understanding of the chromatin transactions in which Hsp90 participates in normal and tumor cells will pave the way for suitable treatment regimens designed for individual pathologies.

\section{Concluding remarks}

Numerous studies in recent years have attributed specific functions to chromatin-associated Hsp90. These range from disassembling transcriptional complexes [14] to maintaining pol II pausing [31]. Almost one-third of all genes - coding and non-coding - appear to be influenced by the chaperone at chromatin. The mechanistic details are emerging gradually, but are difficult to interpret in the light of the inherent complexity of the Hsp90 chaperone network. A unified picture of the chromatin roles of Hsp90 with a systems-level understanding of the participating processes will be an important aim for future analyses. We believe that these findings will lead to a reinterpretation of existing paradigms concerning the role of this chaperone in cancer and evolution.

It is becoming apparent that Hsp90 will be a hub in the epigenetic regulation of gene expression, with specific connections to the promoter-bound apparatus. Conceptually, this presents another case of striking resemblance between nuclear and cellular pathways; for example, the discovery of ubiquitin-dependent extraction of chromatin (UDEC) proteins in the nucleus that are mechanistically similar to endoplasmic reticulum-associated degradation (ERAD) [65]. These cases buttress the point that cells have evolved to reuse the same machinery in different contexts to achieve distinct end results. Thus, our knowledge about the functioning of Hsp90 in cytosol should guide detailed mechanistic experiments underlining critical differences in the two pools of Hsp90.

Few molecules in cells have a position in the molecular network as important and as medically relevant as Hsp90. Novel functions continue to add to its rich repertoire of physiological duties, opening new avenues for therapeutic interventions. Owing to its low amounts in the nuclear compartment, chromatin-associated Hsp90 has received limited attention in past decades. With a suite of biochemically defined activities at promoters, telomeres, and DNA replication hubs charted in the past few years, nuclear Hsp90 will be the focus of intense research in the future. The ramifications of these recent studies reach areas as diverse as epigenetics, medicine, and evolution.

\section{Acknowledgments}

The authors acknowledge financial support by ETH Zurich and EU-NoE 'EpiGeneSys'. They thank Brian Freeman, Gunter Merdes, and anonymous referees for suggestions on the manuscript and Tommy Schlumpf for help with graphics.

\section{References}

1 Taipale, M. et al. (2010) HSP90 at the hub of protein homeostasis: emerging mechanistic insights. Nat. Rev. Mol. Cell Biol. 11, 515-528

2 Neckers, L. et al. (2009) The complex dance of the molecular chaperone Hsp90. Trends Biochem. Sci. 34, 223-226

3 Wandinger, S. et al. (2008) The Hsp90 chaperone machinery. J. Biol. Chem. 283, 18473-18477

4 Jarosz, D.F. et al. (2010) Protein homeostasis and the phenotypic manifestation of genetic diversity: principles and mechanisms. Annu. Rev. Genet. 44, 189-216

5 Porter, J.R. et al. (2010) Discovery and development of Hsp90 inhibitors: a promising pathway for cancer therapy. Curr. Opin. Chem. Biol. 14, 412-420

6 DeZwaan, D.C. and Freeman, B.C. (2010) HSP90 manages the ends. Trends Biochem. Sci. 35, 384-391

7 Sekimoto, T. et al. (2010) The molecular chaperone Hsp90 regulates accumulation of DNA polymerase eta at replication stalling sites in UV-irradiated cells. Mol. Cell 37, 79-89

8 Zelin, E. et al. (2012) The p23 molecular chaperone and GCN5 Acetylase jointly modulate protein-DNA dynamics and open chromatin status. Mol. Cell 48, 459-470

9 Zhao, R. and Houry, W. (2005) Hsp90: a chaperone for protein folding and gene regulation. Biochem. Cell Biol. 83, 703-710

10 Schnaider, T. et al. (1999) Interactions of Hsp90 with histones and related peptides. Life Sci. 65, 2417-2426

11 Csermely, P. et al. (1994) The $90 \mathrm{kDa}$ heat shock protein (Hsp90) induces the condensation of the chromatin structure. Biochem. Biophys. Res. Commun. 202, 1657-1663

12 Morcillo, G. et al. (1993) HSP90 associates with specific heat shock puffs (hsr omega) in polytene chromosomes of Drosophila and Chironomus. Chromosoma 102, 648-659

13 Liu, J. and DeFranco, D.B. (1999) Chromatin recycling of glucocorticoid receptors: implications for multiple roles of heat shock protein $90 \mathrm{Mol}$. Endocrinol. 13, 355-365

14 Freeman, B.C. and Yamamoto, K.R. (2002) Disassembly of transcriptional regulatory complexes by molecular chaperones. Science 296, 2232-2235 
15 Cerchietti, L.C. et al. (2009) A purine scaffold Hsp90 inhibitor destabilizes BCL-6 and has specific antitumor activity in BCL-6dependent B cell lymphomas. Nat. Med. 15, 1369-1376

16 Hung, J-J. et al. (2005) Hsp90alpha recruited by $\mathrm{Sp} 1$ is important for transcription of 12(S)-lipoxygenase in A431 cells. J. Biol. Chem. 280, 36283-36292

17 Moulick, K. et al. (2011) Affinity-based proteomics reveal cancerspecific networks coordinated by Hsp90. Nat. Chem. Biol. 7, 818-826

18 Shaknovich, R. et al. (1992) Conformational activation of a basic helixloop-helix protein (MyoD1) by the C-terminal region of murine HSP90 (HSP84). Mol. Cell. Biol. 12, 5059-5068

19 Tariq, M. et al. (2009) Trithorax requires Hsp90 for maintenance of active chromatin at sites of gene expression. Proc. Natl. Acad. Sci. U.S.A. 106, 1157-1162

20 Hamamoto, R. et al. (2004) SMYD3 encodes a histone methyltransferase involved in the proliferation of cancer cells. Nat. Cell Biol. 6, 731-740

21 Floer, M. et al. (2008) HSP90/70 chaperones are required for rapid nucleosome removal upon induction of the GAL genes of yeast. Proc. Natl. Acad. Sci. U.S.A. 105, 2975-2980

22 Hartson, S.D. and Matts, R.L. (2012) Approaches for defining the Hsp90-dependent proteome. Biochim. Biophys. Acta 1823, 656-667

23 Maloney, A. et al. (2007) Gene and protein expression profiling of human ovarian cancer cells treated with the heat shock protein 90 inhibitor 17-allylamino-17-demethoxygeldanamycin. Cancer Res. 67, 3239-3253

24 Zhao, R. et al. (2005) Navigating the chaperone network: an integrative map of physical and genetic interactions mediated by the Hsp90 chaperone. Cell 120, 715-727

25 Tsaytler, P.A. et al. (2009) Novel Hsp90 partners discovered using complementary proteomic approaches. Cell Stress Chaperones 14, 629-638

26 Sharma, K. et al. (2012) Quantitative proteomics reveals that Hsp90 inhibition preferentially targets kinases and the DNA damage response. Mol. Cell. Proteomics 11, M111.014654

27 Falsone, S.F. et al. (2007) A proteomic approach towards the Hsp90dependent ubiquitinylated proteome. Proteomics 7, 2375-2383

28 Hayashi, M.T. and Masukata, H. (2011) Regulation of DNA replication by chromatin structures: accessibility and recruitment. Chromosoma $120,39-46$

29 Echeverria, P.C. et al. (2009) Nuclear import of the glucocorticoid receptor-hsp90 complex through the nuclear pore complex is mediated by its interaction with Nup62 and importin beta. Mol. Cell. Biol. 29, 4788-4797

30 Akhtar, A. and Gasser, S.M. (2007) The nuclear envelope and transcriptional control. Nat. Rev. Genet. 8, 507-517

31 Sawarkar, R. et al. (2012) Hsp90 globally targets paused RNA polymerase to regulate gene expression in response to environmental stimuli. Cell 149, 807-818

32 Zhang, Z. et al. (2007) Negative elongation factor NELF represses human immunodeficiency virus transcription by pausing the RNA polymerase II complex. J. Biol. Chem. 282, 16981-16988

33 Vozzolo, L. et al. (2010) Gyrase B inhibitor impairs HIV-1 replication by targeting Hsp90 and the capsid protein. J. Biol. Chem. 285, 3931439328

34 Echeverria, P.C. and Picard, D. (2010) Molecular chaperones, essential partners of steroid hormone receptors for activity and mobility. Biochim. Biophys. Acta 1803, 641-649

35 Gano, J.J. and Simon, J.A. (2010) A proteomic investigation of liganddependent HSP90 complexes reveals CHORDC1 as a novel ADPdependent HSP90-interacting protein. Mol. Cell. Proteomics 9, 255-270

36 Boulon, S. et al. (2010) HSP90 and its R2TP/Prefoldin-like cochaperone are involved in the cytoplasmic assembly of RNA polymerase II. Mol. Cell 39, 912-924

37 Mollapour, M. and Neckers, L. (2012) Post-translational modifications of Hsp90 and their contributions to chaperone regulation. Biochim. Biophys. Acta 1823, 648-655

38 Solier, S. et al. (2012) Heat shock protein 90alpha (HSP90alpha), a substrate and chaperone of DNA-PK necessary for the apoptotic response. Proc. Natl. Acad. Sci. U.S.A. 109, 12866-12872

39 Mollapour, M. et al. (2010) Swe1 ${ }^{\text {Wee1 }}$-dependent tyrosine phosphorylation of Hsp90 regulates distinct facets of chaperone function. Mol. Cell 37, 333-343
40 Jamrich, M. et al. (1977) Localization of RNA polymerase in polytene chromosomes of Drosophila melanogaster. Proc. Natl. Acad. Sci. U.S.A. 74, 2079-2083

41 Teves, S.S. and Henikoff, S. (2011) Heat shock reduces stalled RNA polymerase II and nucleosome turnover genome-wide. Genes Dev. 25, 2387-2397

42 Beisel, C. and Paro, R. (2011) Silencing chromatin: comparing modes and mechanisms. Nat. Rev. Genet. 12, 123-135

43 Cernilogar, F.M. et al. (2011) Chromatin-associated RNA interference components contribute to transcriptional regulation in Drosophila. Nature 480, 391-395

44 Miyoshi, T. et al. (2010) A direct role for Hsp90 in pre-RISC formation in Drosophila. Nat. Struct. Mol. Biol. 17, 1024-1026

$45 \mathrm{Ye}$, R. et al. (2012) Cytoplasmic assembly and selective nuclear import of Arabidopsis Argonaute4/siRNA complexes. Mol. Cell 46, 859-870

46 Cook, A.J.L. et al. (2011) A specific function for the histone chaperone NASP to fine-tune a reservoir of soluble H3-H4 in the histone supply chain. Mol. Cell 44, 918-927

47 Deal, R.B. et al. (2010) Genome-wide kinetics of nucleosome turnover determined by metabolic labeling of histones. Science 328, 1161-1164

48 Kaushik, S. and Cuervo, A.M. (2012) Chaperone-mediated autophagy: a unique way to enter the lysosome world. Trends Cell Biol. 22, 407-417

49 Prud'homme, B. et al. (2007) Emerging principles of regulatory evolution. Proc. Natl. Acad. Sci. U.S.A. 104 (Suppl. 1), 8605-8612

50 Hoekstra, H.E. and Coyne, J.A. (2007) The locus of evolution: Evo Devo and the genetics of adaptation. Evolution 61, 995-1016

51 Jarosz, D.F. and Lindquist, S. (2010) Hsp90 and environmental stress transform the adaptive value of natural genetic variation. Science 330 , 1820-1824

52 Sollars, V.et al. (2003) Evidence for an epigenetic mechanism by which Hsp90 acts as a capacitor for morphological evolution. Nat. Genet. 33, $70-74$

53 Ringrose, L. and Paro, R. (2004) Epigenetic regulation of cellular memory by the Polycomb and Trithorax group proteins. Annu. Rev. Genet. 38, 413-443

54 Petruk, S. et al. (2012) TrxG and PcG proteins but not methylated histones remain associated with DNA through replication. Cell 150, 922-933

55 Daxinger, L. and Whitelaw, E. (2012) Understanding transgenerational epigenetic inheritance via the gametes in mammals. Nat. Rev. Genet. 13 , 153-162

56 Trepel, J. et al. (2010) Targeting the dynamic HSP90 complex in cancer. Nat. Rev. Cancer 10, 537-549

57 Neckers, L. and Workman, P. (2012) Hsp90 molecular chaperone inhibitors: are we there yet? Clin. Cancer Res. 18, 64-76

58 Jhaveri, K. et al. (2012) Advances in the clinical development of heat shock protein 90 (Hsp90) inhibitors in cancers. Biochim. Biophys. Acta 1823, 742-755

59 Smith, E. et al. (2011) The super elongation complex (SEC) and MLL in development and disease. Genes Dev. 25, 661-672

60 Mendillo, M.L. et al. (2012) HSF1 drives a transcriptional program distinct from heat shock to support highly malignant human cancers. Cell 150, 549-562

61 Rao, R. et al. (2008) HDAC6 inhibition enhances 17-AAG-mediated abrogation of hsp90 chaperone function in human leukemia cells. Blood 112, 1886-1893

62 Piper, P.W. and Millson, S.H. (2011) Mechanisms of resistance to Hsp90 inhibitor drugs: a complex mosaic emerges. Pharmaceuticals 4, 1400-1422

63 Kang, B.H. et al. (2007) Regulation of tumor cell mitochondrial homeostasis by an organelle-specific Hsp90 chaperone network. Cell $131,257-270$

64 Kose, S. et al. (2012) Hikeshi, a nuclear import carrier for Hsp70s, protects cells from heat shock-induced nuclear damage. Cell 149, 578-589

65 Dantuma, N.P. and Hoppe, T. (2012) Growing sphere of influence: Cdc48/p97 orchestrates ubiquitin-dependent extraction from chromatin. Trends Cell Biol. 22, 483-491

66 Prodromou, C. (2012) The 'active life' of Hsp90 complexes. Biochim. Biophys. Acta 1823, 614-623

67 Didenko, T. et al. (2012) Hsp90 structure and function studied by NMR spectroscopy. Biochim. Biophys. Acta 1823, 636-647 
$68 \mathrm{Li}$, J. et al. (2012) The Hsp90 chaperone machinery: conformational dynamics and regulation by co-chaperones. Biochim. Biophys. Acta 1823, 624-635

69 Johnson, J.L. (2012) Evolution and function of diverse Hsp90 homologs and cochaperone proteins. Biochim. Biophys. Acta 1823, 607-613

70 Makhnevych, T. and Houry, W.A. (2012) The role of Hsp90 in protein complex assembly. Biochim. Biophys. Acta 1823, 674-682

71 Carthew, R.W. and Sontheimer, E.J. (2009) Origins and mechanisms of miRNAs and siRNAs. Cell 136, 642-655

72 Sawarkar, R. and Paro, R. (2010) Interpretation of developmental signaling at chromatin: the Polycomb perspective. Dev. Cell 19, 651-661

73 Bantignies, F. and Cavalli, G. (2006) Cellular memory and dynamic regulation of polycomb group proteins. Curr. Opin. Cell Biol. 18, 275-283

74 Schuettengruber, B. et al. (2011) Trithorax group proteins: switching genes on and keeping them active. Nat. Rev. Mol. Cell Biol. 12, 799-814

75 Skarra, D.V. et al. (2011) Label-free quantitative proteomics and SAINT analysis enable interactome mapping for the human Ser/Thr protein phosphatase 5. Proteomics 11, 1508-1516

76 Te, J. et al. (2007) Novel subunits of the mammalian Hsp90 signal transduction chaperone. J. Proteome Res. 6, 1963-1973

77 Wang, X. et al. (2010) Geldanamycin-induced PCNA degradation in isolated Hsp90 complex from cancer cells. Cancer Invest. 28, 635-641

78 McClellan, A.J. et al. (2007) Diverse cellular functions of the Hsp90 molecular chaperone uncovered using systems approaches. Cell 131, 121-135
79 Song, D. et al. (2008) Antitumor activity and molecular effects of the novel heat shock protein 90 inhibitor, IPI-504, in pancreatic cancer. Mol. Cancer Ther. 7, 3275-3284

80 Millson, S.H. et al. (2005) A two-hybrid screen of the yeast proteome for Hsp90 interactors uncovers a novel Hsp90 chaperone requirement in the activity of a stress-activated mitogen-activated protein kinase, Slt2p (Mpk1p). Eukaryot. Cell 4, 849-860

81 Core, L.J. and Lis, J.T. (2008) Transcription regulation through promoter-proximal pausing of RNA polymerase II. Science 319, 1791-1792

82 Nechaev, S. and Adelman, K. (2011) Pol II waiting in the starting gates: regulating the transition from transcription initiation into productive elongation. Biochim. Biophys. Acta 1809, 34-45

83 Levine, M. (2011) Paused RNA polymerase II as a developmental checkpoint. Cell 145, 502-511

84 Adelman, K. and Lis, J.T. (2012) Promoter-proximal pausing of RNA polymerase II: emerging roles in metazoans. Nat. Rev. Genet. 13, 720-731

85 Queitsch, C. et al. (2002) Hsp90 as a capacitor of phenotypic variation. Nature 417, 618-624

86 Rutherford, S.L. and Lindquist, S. (1998) Hsp90 as a capacitor for morphological evolution. Nature 396, 336-342

87 Sangster, T.A. et al. (2004) Under cover: causes, effects and implications of Hsp90-mediated genetic capacitance. Bioessays 26, 348-362

88 Specchia, V. et al. (2010) Hsp90 prevents phenotypic variation by suppressing the mutagenic activity of transposons. Nature 463, 662-665

89 Sawarkar, R. and Paro, R. (2010) Heat shock protein 90: a capacitor or a mutator? J. Biosci. 35, 163-165 